\title{
A rare case report of neonatal calcinosis cutis induced by distant and delayed extravasation of intravenous calcium gluconate
}

\author{
Kwang Hyeon Ahn, Eun Soo Park \\ Department of Plastic and Reconstructive Surgery, Soonchunhyang University Bucheon Hospital, Bucheon, Korea
}

\begin{abstract}
A 3,480 g male neonate showed tachypnea symptom with a serum ionized calcium level of $0.66 \mathrm{mmol} / \mathrm{L}$ by routine clinical analysis. He was injected calcium gluconate intravenously through femoral vein catheter to treat the hypocalcemia. On second day after the injection, he started to show erythema in the flank area. The lesion became firm and changed into whitish crust consist of small crystals. Abdominal X-ray and ultrasonography showed the accumulation of calcium deposit in the subcutaneous layer of the lesion. Surgical debridement was performed to remove the crust with calcium deposit and acellular fish skin graft rich in omega-3 (Kerecis) was applied to the defect site for secondary intention of the defect wound. After 2 months, the skin and soft tissue defect were fully covered with healthy normal skin without depression or contracture. This report is a first case of iatrogenic calcinosis cutis without extravasation symptom.
\end{abstract}

Keywords Piscine skin acellular matrix / Kerecis / Calcinosis cutis / Infant, newborn

\author{
Correspondence: Eun Soo Park \\ Department of Plastic and \\ Reconstructive Surgery, \\ Soonchunhyang University Bucheon \\ Hospital, 170 Jomaru-ro, Wonmi-gu, \\ Bucheon 14584, Korea \\ Tel: +82-32-621-5319 \\ Fax: +82-32-621-5016 \\ E-mail: peunsoo@schmc.ac.kr
}

This work was supported by the Soonchunhyang University Research Fund.

Received: October 9, $2020 \bullet$ Revised: June 17, $2021 \bullet$ Accepted: July 7, 2021

pISSN: 2234-6163 • elSSN: 2234-6171 • https://doi.org/10.5999/aps.2020.01942 • Arch Plast Surg 2021;48:641-645

\section{INTRODUCTION}

Hypocalcemia is a frequently observed clinical and laboratory abnormality in neonates. Ionic calcium is crucial for many biochemical processes including blood coagulation, neuromuscular excitability, cell membrane integrity, and many of the cellular enzymatic activities [1]. Hypocalcemia is defined as total serum calcium of less than $7 \mathrm{mg} / \mathrm{dL}(1.75 \mathrm{mmol} / \mathrm{L})$ or ionized calcium less than $4 \mathrm{mg} / \mathrm{dL}(1 \mathrm{mmol} / \mathrm{L})$ in preterm infants and less than $8 \mathrm{mg} / \mathrm{dL}(2 \mathrm{mmol} / \mathrm{L}$; total) or $<1.2 \mathrm{mmol} / \mathrm{L}$ (ionic) in term neonates [2]. Neonatal hypocalcemia can cause neuromuscular irritability, prolonged QT interval, and furthermore, in severe hypocalcemia, it can lead to decreased contractibility and heart failure. So early management of hypocalcemia is im- portant to prevent these complications. In the case of patient with neuromuscular irritability, prompt management with intravenous calcium is required [3]. Sometimes, during the intravenous injection, the extravasation of calcium gluconate can occur. The symptoms are marked by swelling and erythema, and these symptoms usually resolve with conservative treatment [4-6]. In case of severe symptoms like soft tissue necrosis or infection with local calcification, it is called calcinosis cutis. Presentation of calcinosis cutis after injection of intravenous calcium gluconate is very rare, and they are only two reports of this disease in the orthopedic literature [7]. This report is about this rare case of a neonate with calcinosis cutis after calcium gluconate injection for hypocalcemia in a neonate. The approval of the Institutional Review Board of Soonchunhyang University Bucheon 
Hospital (IRB No. 2021-04-001) was obtained for review of the patient record. The patients' legal guardian provided informed consent for the publication of clinical case and the accompanying patients' photographs.

\section{CASE}

A 3,480 g male neonate was delivered at 40 weeks of gestation and labor by vaginal birth. Mother was 42 years old and have no past medical history. She did not use any medication during the pregnancy period and had taken scheduled prenatal examinations. There was no family history regarding congenital anomaly.

The child's Apgar score was 8 at 1 minute and 10 at 5 minutes. He was stable until the 48 hours after birth when he showed tachypnea symptom. The respiratory rate was continuously checked over 50 per minute. He was transferred to author's hospital for neonatal intensive care unit (NICU) management and the serum ionized calcium level was $0.66 \mathrm{mmol} / \mathrm{L}$ by routine clinical analysis. The hospital's normal value of calcium level was 1.09 to $1.30 \mathrm{mmol} / \mathrm{L}$. Next, follow-up routine analysis showed ionized calcium level of $0.45 \mathrm{mg} / \mathrm{dL}$ and he started to show tetanus symptoms. The patient was diagnosed neonatal hypocalcemia, and the he was injected calcium gluconate intravenously through femoral vein catheter. The initial loading dose was $600 \mathrm{mg}$ of calcium gluconate, and another $2.5 \mathrm{~g}$ of calcium gluconate was additionally injected. In second day after the injection, he started to show $5 \times 2 \mathrm{~cm}$ size of erythema in the flank area (Fig. 1A), and the erythema started to change into firm and whitish crust from the margin to center.

We tried to prevent infection of the wound by dressing the wound with antiseptic ointment, and tried not to dry up the wound with the hydrocellular foam dressing material. He was discharged on 9 days of admission, and routinely visited plastic surgery outpatient department for the management of the flank wound. The skin and subcutaneous tissue of the flank wound changed into total whitish crust consist of small crystals (Fig. 1B). Abdominal X-ray and ultrasonography showed accumulation of calcium deposit in the subcutaneous lesion of the flank (Fig. 2). He was finally diagnosed with calcinosis cutis following injection of calcium gluconate. When the crust became completely delineated, surgical debridement was performed (Fig. 3A). Careful dissection was done to minimize the soft tissue injury and the deep margin of the crust was only limited in subcutaneous layer. After the debridement, the defect size was $4 \times 3 \mathrm{~cm}$. There was no exposure of fascia, and muscle layer. Acellular fish skin graft rich in omega-3 (Kerecis, Arlington, VA, USA) was applied to the defect site for secondary intention of the wound (Fig. 3B). The Kerecis was cut according to the wound size, and soaked in $0.9 \%$ normal saline for 1 minute for hydration.

Then it was applied to the wound, and covered with hydrocellular foam (Allevyn Gentle Border; Smith \& Nephew, London, UK). The patient visited outpatient department two times per week. At each visit, the wound was cleaned and debrided, the wound size was photodocumented. New omega-3 matrix was re-applied to the wound.

The wound size diminished day by day with marginal epithelization (Fig. 3C). There was no infection sign during the treatment. After 2 months, the defect was fully covered with healthy normal skin. There was no depression or contracture at the wound site. There was only pigmentation in the scar. The pa-

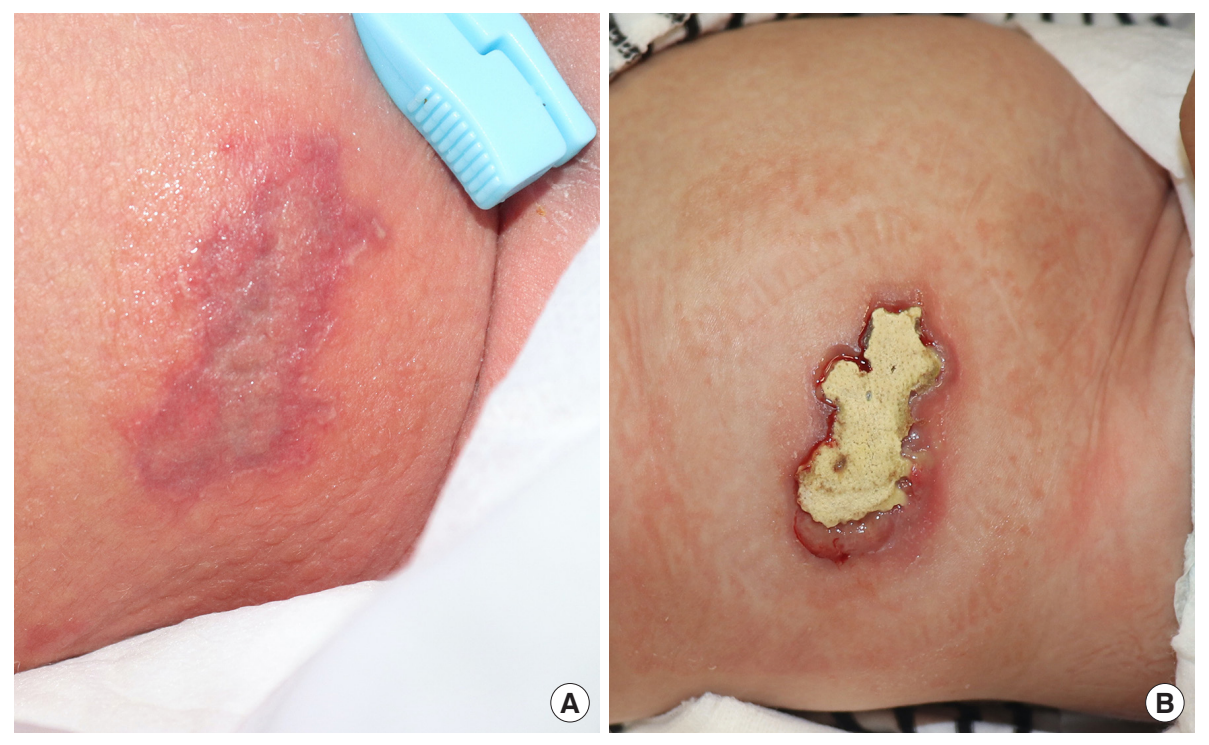

Fig. 1. Initial photographic findings of calcinosis cutis at flank area. (A) The patient had erythema in the flank area and its initial size was $5 \times 2$ $\mathrm{cm}$. (B) The erythema became firm and changed into whitish crust consist of small crystals. 

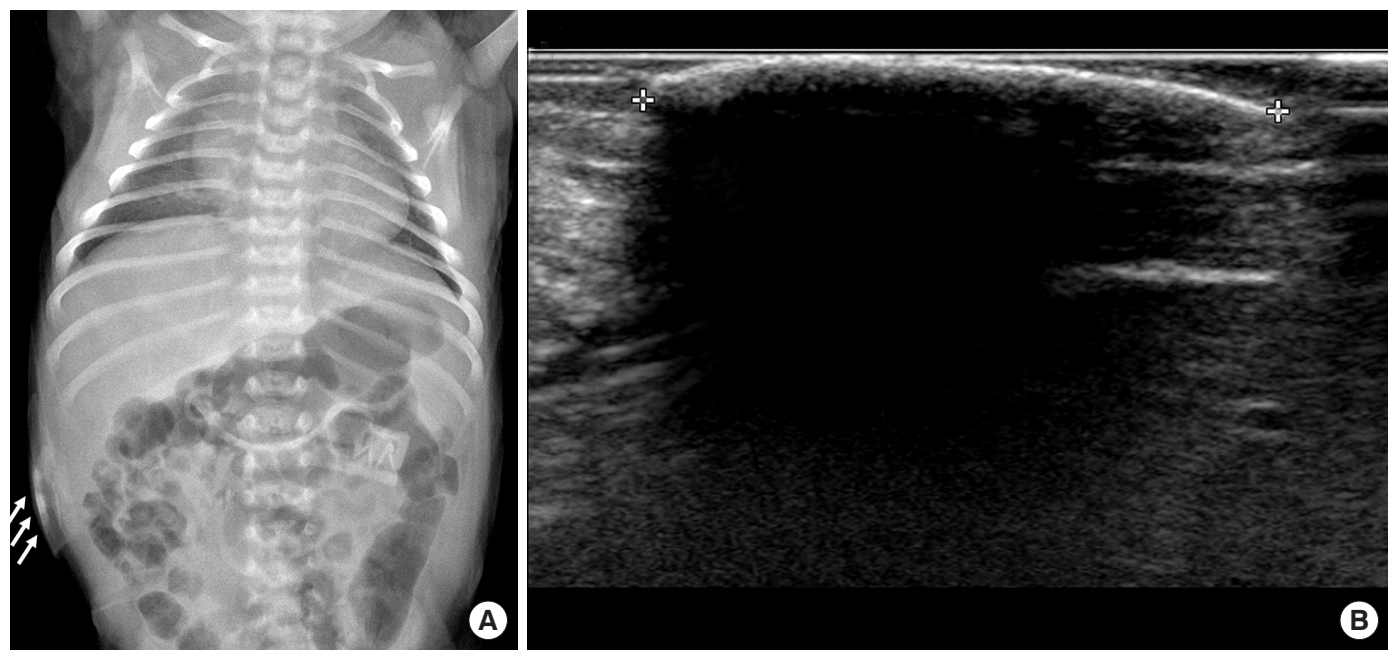

Fig. 2. Radiographic findings of calcinosis cutis. Abdominal X-ray (A) and ultrasonography (B) showed accumulation of calcium deposit in the subcutaneous lesion of the flank (white arrows).
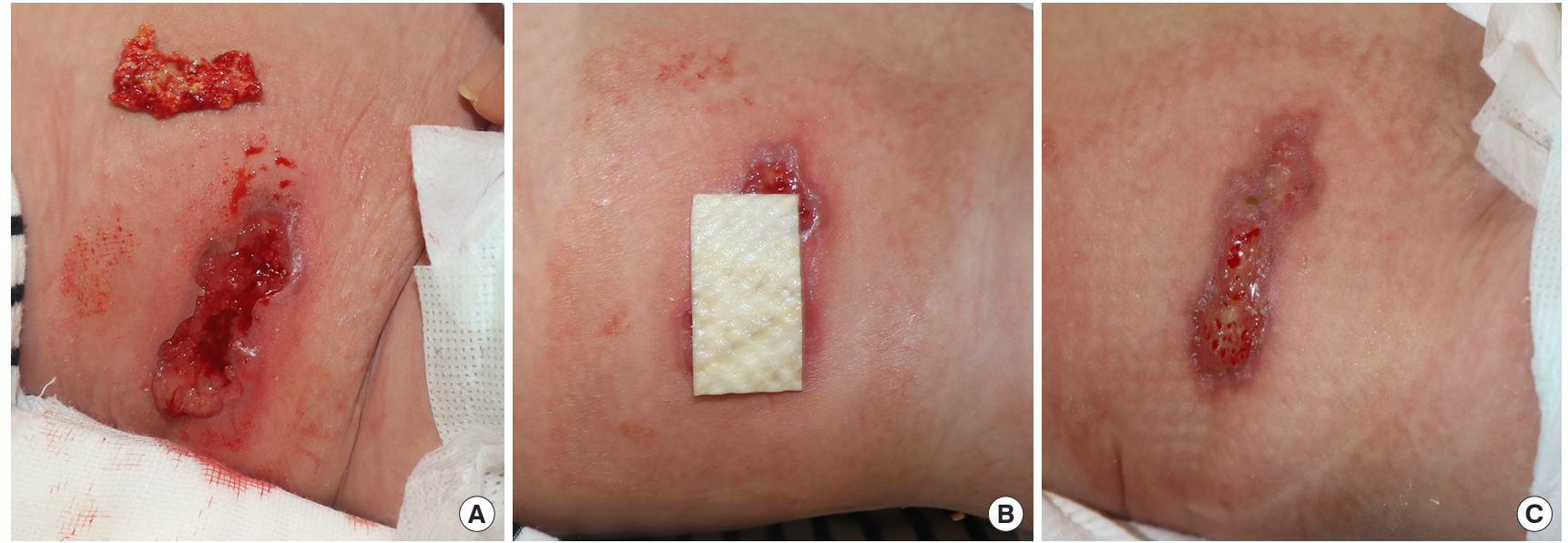

Fig. 3. Debridement and Kerecis application. Surgical debridement was performed (A) and acellular fish skin graft rich in omega-3 (Kerecis) was applied to the defect site for secondary intention of the wound (B). (C) The wound size diminished day by day with marginal epithelization.

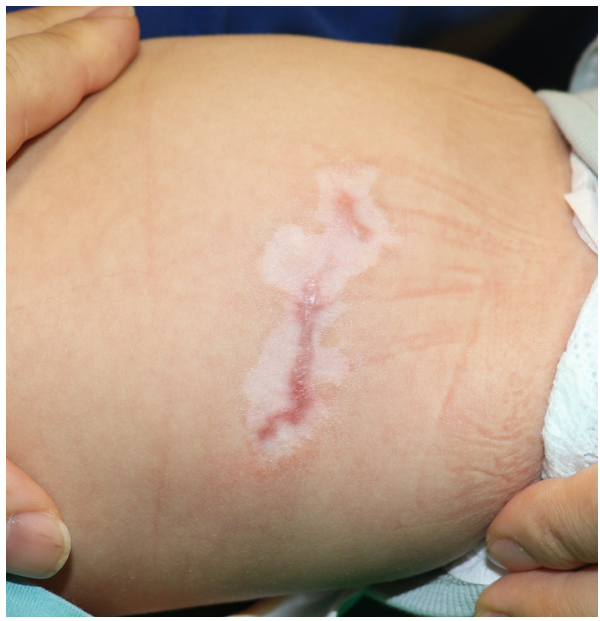

Fig. 4. Follow-up photographic finding after 4 months. The patient continued to follow up for the wound observation and there was no more deposit of calcification since the complete healing. tient continued to follow up for the wound observation and there was no more deposit of calcification since the complete healing (Fig. 4). The follow-up X-ray showed no more calcification deposit in the flank area.

\section{DISCUSSION}

The first two case about the complication of calcium gluconate injection were reported in 1936 in the medical literature $[8,9]$. In these cases, the intramuscular injection was pointed as a risk factor, so since then, the intravenous injection was recommended to reduce iatrogenic complication [10]. Sometimes, intravenous injection may be extravasated, which means leakage of injection out of the vein to the surrounding soft tissue. This leakage result to accumulation of calcium crystal in the skin and soft tissue of the patient, leading infection and necrosis of the tissue. 
These condition is named calcinosis cutis.

The type of calcinosis cutis are categorized into four categories: dystrophic, metastatic, idiopathic, and iatrogenic [11]. Dystrophic type is calcification resulted from local tissue infection or diseased tissues [12]. Metastatic calcification is result of abnormal calcium or phosphate metabolism caused by systemic disease [12]. Idiopathic calcification is rarely occurs, and any process causing calcification can result secondary ossification [13]. Iatrogenic type is accumulation of calcium crystal after injection of calcium gluconate [14].

Extravasation is damage caused by the efflux of solutions from a vessel into surrounding tissue spaces during intravenous infusion [15]. In the prior cases about the calcinosis cutis following extravasation of calcium gluconate, the wound is near the injection site [7]. Sonohata et al. [7] reported a case of calcinosis cutis in the dorsum of wrist and the injection and extravasation site was the dorsum of the affected hand. In our case, the injection site was right dorsum of the feet, and the extravasation and calcification occurred at the flank of the patient. It is a rare case of calcinosis cutis as the deposit site is remote from the injection site of calcium gluconate. As typical iatrogenic calcinosis cutis occurs following extravasation, it was difficult to diagnose this atypical patient. We first assumed the wound as pressure sore by the inappropriate position change in NICU. However, the wound become whitish from the margin, and the whitish crust was consist of calcium-like crystal. So we all reviewed the medical record of the patient from the beginning, and found the use of calcium gluconate for management of hypocalcemia. This case is important case of calcinosis cutis, as iatrogenic calcification can occur anywhere in the body, unlike the typical extravasation cases. So clinicians should remind not to neglect calcinosis cutis even though there is no extravasation symptom around the injection site of calcium gluconate.

In this case, we used Kerecis to promote secondary intention of the wound. Kerecis is acellular fish skin graft rich in omega-3. This fish skin graft is composed of important components of extracellular matrix structural proteins including glycosaminoglycan, proteoglycan, fibronectin, and growth factors [16]. These components promote granulation and epithelization of the wounds [17]. Also, in fish skin graft, there is additional benefits of omega-3 lipids. Omega-3 fatty acids help wound healing with anti-inflammatory effect and are resistant to bacterial colonization $[18,19]$. With combination of extracellular matrix component and omega-3 lipids, the Kerecis provide nutrient wound environment for epithelization. The authors also observed the dynamic improvement of the affected wound with use of this acellular fish skin graft. There was fast granulation of the tissue, and there was no infection sign until the 2 months of total epithelization. Another advantage of piscine skin acellular matrix was the convenience of dressing. The patient do not need to change the dressing daily. Just visiting the outpatient department one or two times per week is enough. Especially in this case, as the patient was infant, simplifying the dressing method with the Kerecis was better than other dressing methods for both doctor and patient.

We describe a rare and untypical case of neonate with calcinosis cutis lesion on flank, following the extravasation of distantly injected intravenous calcium gluconate. Several case reports were documented in dermatology and pediatric literature about the calcinosis cutis following the immediate extravasation of the calcium gluconate in injection site, but there was no case of extravasation of distantly injected intravenous calcium gluconate. This report is a first case of iatrogenic calcinosis cutis with distant and delayed extravasation of calcium gluconate. Clinicians always remind there can be iatrogenic calcinosis cutis with the distant and delayed extravasation of the injection, and careful examination of the wound and review of medical history may be useful for diagnosis of this disease. Also, the Kerecis provided fast healing and great aesthetic result in this patient.

\section{NOTES}

\section{Conflict of interest}

No potential conflict of interest relevant to this article was reported.

\section{Ethical approval}

The study was approved by the Institutional Review Board of Soonchunhyang University Bucheon Hospital (IRB No. 202104-001) and performed in accordance with the principles of the Declaration of Helsinki. Written informed consent was obtained.

\section{Patient consent}

The patient's legal guardian provided written informed consent for the publication and the use of the patient's images.

\section{Author contribution}

Conceptualization: KH Ahn, ES Park. Data curation: KH Ahn. Formal analysis: KH Ahn. Methodology: ES Park. Project administration: ES Park. Visualization: ES Park. Writing - original draft: KH Ahn. Writing - review \& editing: ES Park.

\section{ORCID}

Kwang Hyeon Ahn https://orcid.org/0000-0002-3297-2336

Eun Soo Park https://orcid.org/0000-0003-2966-9122 


\section{REFERENCES}

1. Jain A, Agarwal R, Sankar MJ, et al. Hypocalcemia in the newborn. Indian J Pediatr 2010;77:1123-8.

2. Oden J, Bourgeois M. Neonatal endocrinology. Indian J Pediatr 2000;67:217-23.

3. Cooper MS, Gittoes NJ. Diagnosis and management of hypocalcaemia. BMJ 2008;336:1298-302.

4. Tuncer S, Aydin A, Erer M. Extravasation of calcium solution leading to calcinosis cutis surrounding the dorsal cutaneous branch of the ulnar nerve. J Hand Surg Br 2006;31: 288-9.

5. Goldminz D, Barnhill R, McGuire J, et al. Calcinosis cutis following extravasation of calcium chloride. Arch Dermatol 1988;124:922-5.

6. Wolfe MS, North ER. Extravasation of injected calcium solution leading to calcifications in the upper extremity of the neonate: report of a case. J Bone Joint Surg Am 1983;65: 558-9.

7. Sonohata M, Akiyama T, Fujita I, et al. Neonate with calcinosis cutis following extravasation of calcium gluconate. J Orthop Sci 2008;13:269-72.

8. Tumpeer IH. Calcium deposit following therapeutic injections in tetany of the newborn. Arch Pediatr 1936;53:21523.

9. Von Hofe FH, Jennings RE. Calcium deposition following the intramuscular administration of calcium gluconate: report of a case in a newborn infant.J Pediatr 1936;8:348-51.

10. Puvabanditsin S, Garrow E, Titapiwatanakun R, et al. Severe calcinosis cutis in an infant. Pediatr Radiol 2005;35:539-42.

11. Caksen H, Odabas D. An infant with gigantic subcutaneous calcium deposition following extravasation of calcium gluconate. Pediatr Dermatol 2002;19:277-9.

12. Touart DM, Sau P. Cutaneous deposition diseases: part II. J Am Acad Dermatol 1998;39(4 Pt 1):527-44.

13. Walsh JS, Fairley JA. Calcifying disorders of the skin. J Am Acad Dermatol 1995;33(5 Pt 1):693-706.

14. Ramamurthy RS, Harris V, Pildes RS. Subcutaneous calcium deposition in the neonate associated with intravenous administration of calcium gluconate. Pediatrics 1975;55: 802-6.

15. Al-Benna S, O'Boyle C, Holley J. Extravasation injuries in adults. ISRN Dermatol 2013;2013:856541.

16. Armstrong RB, Nichols J, Pachance J. Punch biopsy wounds treated with Monsel's solution or a collagen matrix: a comparison of healing. Arch Dermatol 1986;122:546-9.

17. Baldursson BT, Kjartansson H, Konradsdottir F, et al. Healing rate and autoimmune safety of full-thickness wounds treated with fish skin acellular dermal matrix versus porcine small-intestine submucosa: a noninferiority study. Int J Low Extrem Wounds 2015;14:37-43.

18. McDaniel JC, Belury M, Ahijevych K, et al. Omega-3 fatty acids effect on wound healing. Wound Repair Regen 2008; 16:337-45.

19. Trinh TT, Dunschede F, Vahl CF, et al. Marine omega3 wound matrix for the treatment of complicated wounds. Phlebologie 2016;45:93-8. 